\title{
The main directions of increasing the reliability of gas distribution systems in high-rise districts
}

\author{
Viktor Zhila ${ }^{1}$, and Elena Solovyeva ${ }^{1 *}$ \\ ${ }^{1}$ Moscow State University of Civil Engineering, Yaroslavskoe shosse, 26, Moscow, 129337, Russia
}

\begin{abstract}
The main source of energy in the economy is gas fuel. The specific weight of natural gas in the fuel and energy balance is about $50 \%$. the main requirement for the gas distribution system is to ensure the reliability, safety of this system. One of the ways to improve the reliability of gas supply systems is to use the reliability of automatic equipment to determine the gas contamination of premises. Based on the analysis of calculation formulas, an algorithm for the hydraulic calculation of gas pipelines is compiled. Based on the characteristics of gas distribution systems, it is advisable to apply such a solution, when gas supply of domestic consumers, boiler houses of municipal agricultural enterprises is carried out from the distribution network of high and medium pressure through autonomous combined pressure regulators installed in areas of high-rise buildings.
\end{abstract}

\section{Introduction}

The history of the Russian gas industry originates in 1899, when the first gas lanterns were lit in St. Petersburg. By the end of the XIX century, except for the capital, were gasified Moscow, Kiev, Kharkov, Rostov-on-Don, Odessa, Riga, Vilno, Tver and Kazan [1]. Gas began to be used for the needs of industry - melting, metal hardening, fabrics singeing. In $\mathrm{Baku}$, the largest oil-producing region of the Russian Empire, in 1917, 33 million $\mathrm{m}^{3}$ of gas was used.

In 1948, the Main Directorate for Natural Gas Production was established. In 1956, it was transformed into the Main Directorate of the Gas Industry, and then into the State Committee for the Gas Industry. A decade later the Ministry of the Gas Industry started its work. In the second half of the 1970s, gas production in the USSR increased by 4.8 times, and in 1984 the country came out on top in the world in terms of its production - about 590 billion $\mathrm{m}^{3}$ per year. The maximum record in the territory of the former Soviet Union ( over 815 billion $\mathrm{m}^{3}$ ) was recorded in 1992. In the 1960s, in the north of the Tyumen region gas areas were dissolved. In 1970-1980 in Western Siberia, the largest natural gas fields: Vyngapurovskoye, Medvezhye, Urengoiskoye, Yamburgskoye, were discovered, huge gas reserves were discovered on the Yamal Peninsula. Super heavy gas mains were laid in the central and western regions of the country.

\footnotetext{
* Corresponding author solovyva@mail.ru
} 
In conditions when the Soviet raw material resources were thousands of kilometers away from the final consumption places, their rational use could be achieved only if all the resources were combined into a single gas supply network. It was created as a single organism that reacts momentarily to minor changes in any of its links, including operating gas and gas condensate fields [2], gas mains with installed compressor stations, underground gas storage facilities, gas processing plants and distribution stations.

The presence of the Unified Gas Supply System made it possible to optimize the gas production levels in the regions, rationally generate gas transportation flows, reliably and uninterruptedly supply gas to most areas of the country, and also export gas on a large scale. By 1989 , the share of gas in the country's fuel balance reached $38.9 \%$ and approached the share of oil (39\%), significantly exceeded the share of peat $(1.7 \%)$.

Gas routes from Russia to Europe are promising, since Russia is the largest supplier of "blue fuel", and Europe is the largest consumer. The main source of energy for all sectors of the economy is fuel resources [3]. Gas, oil and coal are the most important in the fuel industry. According to geologists in the bowels of Russia, more than $32 \%$ of the world's gas reserves, $13 \%$ of oil and about $30 \%$ of coal are concentrated.

The specific weight of natural gas in the fuel and energy balance of the country is about $50 \%$. Such a share of natural gas in the fuel and energy balance can be considered rational due to the best ecological characteristics of gas, compared to coal and fuel oil. However, the main orientation of the economy on one type of energy carrier negatively affects the economic security of the country and increases the burden on the gas industry.

In the industrially developed countries of the West, the share of natural gas in the fuel and energy balance does not exceed $30 \%$, which is provided by state regulation of energy prices. The price of gas in the domestic market of Russia is about 3 times lower than the price of fuel oil and 46 times lower than the price of coal. In market conditions, "cheap" natural gas inevitably displaces other fuels, reducing their share in the fuel and energy balance of Russia [4]. One of the two main reasons for the decline in the extraction of oil and gas is the natural depletion of the raw materials base at a certain stage of operation. It was already quite evident in the 1980s. In the 1990s, the progressive depletion of non-renewable hydrocarbon resources and the decline in investment efficiency in the explored work were complicated by a sharp reduction in investment. At the beginning of the 1990s, gas production was declining. However, even in these conditions, the gas industry is the most stable industry in the Russian economy. Washington is actively opposed to the construction of the Nord Stream-2 gas pipeline and is trying to squeeze Russia out of the European fuel market. The prices of liquefied natural gas exceed the cost of pipeline gas by $25 \%$. Last year, the average cost of a thousand cubic meters of Russian "blue" fuel was \$167. This year, Gazprom predicts \$ 187. The price of American liquefied gas for Europeans is approximately $\$ 240$ per thousand cubic meters. For the first half of 2017, Gazprom's exports to Europe increased by $12.3 \%$ or 10.5 billion cubic meters. In total, 95.7 billion cubic meters of gas were supplied from Russia under EU contracts. The capacity of one standard tanker is 155 million cubic meters of liquefied natural gas or 95 million cubic meters of natural gas. That is a thousand times less than the volume that is supplied by Russian pipelines. The US will have to send several hundreds of gas carriers to Europe every year. A lot of time will be spent on transportation, liquefaction and regasification (conversion of LNG from liquefied to gaseous state).

\section{Methods}

Over time, there are changes in technical and economic conditions for the development of the Russian gas economy. The main requirement for the gas distribution system is to ensure the reliability, safety and effectiveness of the action in high-rise districts $[5,6,7]$. 
One of the ways to improve the reliability of gas consumption systems is the use of modern automatic equipment to determine the gas content of buildings and gas accumulation sites [8]. Another important factors ensuring reliability and safety is the use of modern means and methods for assessing the gas content of cellar, wells, mines, reservoirs, and soil (Fig. 1).

Various gas analyzers in the explosion-proof housing are in demand, because their use even in a gassed environment does not lead to ignition of the gas-air mixture. If a leak is detected, workers must immediately notify the emergency dispatch service and, before the brigade arrives, take measures to ensure control over the place of the alleged leak and the inadmissibility of using open flames. It is necessary to establish causes of gas leaks: joint breakage, pipe corrosion, mechanical damage, not density in flange connections. The complexity of finding a gas leak from an underground gas pipeline is due to the inaccessibility of the gas pipeline for visual observation and its considerable extent. This circumstance makes it impossible to completely verify it, and consequently the search for a gas leak involves knowledge of the gas pipeline device and the most likely places of leakage $[9,10]$. For this purpose, maps should be developed, where welded joints, the location of inspection wells and cellars, carpets, places with an expected drop in altitude and a change in the slope of the gas pipeline, types of valves should be marked [11].

\section{Results}

Results of the use of formulas for the hydraulic calculation of distribution gas networks.

The main part of the distribution pipelines is made up of steel pipes. The share of polyethylene is about $10 \%$. It is necessary to increase the length of laying polyethylene pipelines in high-rise districts, as they have a number of positive properties.

In this case, polyethylene pipes have a number of positive properties [12]:

- high chemical resistance;

- elasticity;

- flexibility of the pipeline - the minimum radius of bending is 25 pipe diameters at 20 degrees;

- high resistance to abrasion due to smooth and elastic inner wall of the pipe;

- lightness, reliability, quick installation of polyethylene pipes;

- comparative cheapness of polyethylene pipes;

- durability (minimum 50 years).

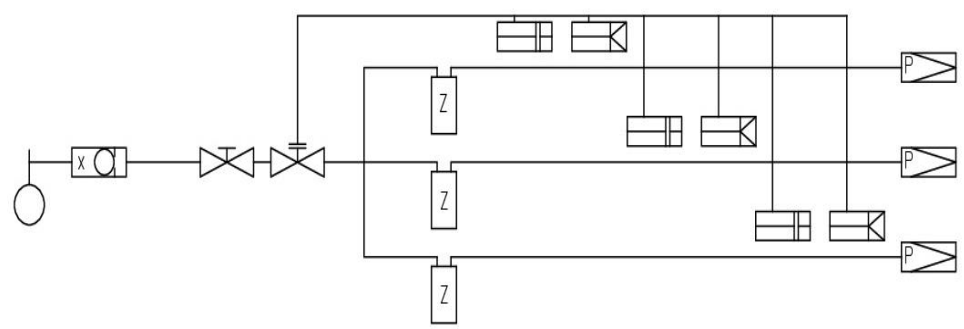

Fig. 1. Apartment house with gas equipment on floors or apartments 


$\begin{array}{ll}\text { - The main isolating device (tap) } & \text { - Gas pressure regulator (regulator) } \\ \text { - Shutoff valve (controller) for gas consumption } & \text { - Counter device } \\ & \text { - Gas pollution detector } \\ & \text { - Fire alarm device } \\ & \text { - Electromagnetic valve } \\ \end{array}$

Characteristic property of polyethylene pipes is the time dependence of strength on temperature and load. In this regard, the life of polyethylene pipes is established on the basis of the predicted values of the long-term strength (MRS), determined by the graph of the longterm strength based on the results of short-term testing of samples in laboratory conditions according to GOST 24157, ISO 9080. During operation, the pipeline is subjected to additional loads - groundwater recharge, soil punching, backfilling, forces caused by a change in pipeline temperature. These and other external factors are taken into account by the safety factor $c=2.5$ when calculating the working pressure in the gas pipeline (MOP) for a period of 50 years $[4,13]$.

$$
\mathrm{MOP}=\frac{2 M R S}{c(S D R-1)} .
$$

For the hydraulic calculation of polyethylene gas pipelines, certain features must be taken into account. Taking into account the gas compressibility, the basic formula has for medium and high pressure networks the following form [14]:

$$
P_{b}^{2}-P_{\mathrm{e}}^{2}=1,62 \lambda \frac{Q_{0}^{2}}{d^{5}} \rho_{0} P_{0} \frac{T}{T_{0}} l
$$

where $P_{b}$ and $\mathrm{P}_{\mathrm{e}}-$ absolute pressure respectively at the beginning and end of the section, $\mathrm{MPa} ; P_{0}-$ $0,0101325 \mathrm{MPa} ; \lambda$ - coefficient of hydraulic friction; $l$ - length of section, $\mathrm{m} ; d$-internal diameter of a gas pipeline; $\rho_{0}$ - gas density under normal conditions; $Q_{0}$ - gas consumption; $T$ - gas temperature, ${ }^{0} \mathrm{C}$. The coefficient of hydraulic friction $\lambda$ depends on the flow regime of the hydraulic smoothness of the pipe:

- laminar flow of gas $\operatorname{Re}<2000$

$$
\lambda=\frac{64}{\operatorname{Re}}
$$

- critical mode: when $\mathrm{Re}=2000-4000$

$$
\lambda=0,0025 \mathrm{Re}^{0.333} ;
$$

- when $\operatorname{Re}>4000$ depending on the hydraulic smoothness of the gas pipeline. Conditions for the hydraulic smoothness of a pipe are determined by the inequality:

$$
\lambda=0,11\left(\frac{n}{d}+\frac{68}{\operatorname{Re}}\right)^{0.25},
$$


where $n$ - equivalent roughness for polyethylene pipes;

$$
\begin{gathered}
P_{b}^{2}-P_{\mathrm{e}}^{2}=1.62 \cdot 0,11\left(\frac{n}{d}+\frac{68}{\mathrm{Re}}\right)^{0.25} \quad \cdot \frac{Q_{0}^{2}}{d^{5}} \rho_{0} P_{0} \frac{T}{T_{0}} l=0.1782\left(\frac{0,0007}{d}+\frac{68}{\frac{4 Q_{0} \rho_{0}}{\pi \mu d}}\right)^{0.25} \times \\
\times \frac{Q_{0}^{2}}{d^{5}} \rho_{0} P_{0} \frac{273+17}{273} l .
\end{gathered}
$$

In this case gas is an incompressible fluid for low pressure networks and is an ideal gas for networks of all medium and high pressure categories.

$$
\begin{gathered}
P_{b}^{2}-P_{\mathrm{e}}^{2}=0,1782\left(\frac{0,0007}{\mathrm{~d}}+\frac{68 \cdot 10.9 \cdot 10^{-6} d \cdot 3.14}{4 \cdot 0.748 Q_{0}}\right)^{0.25} \cdot \frac{Q_{0}^{2}}{d^{5}} \cdot 0.478 \cdot 101300 \cdot 1.06 l \\
P_{b}^{2}-P_{\mathrm{e}}^{2}=0.014313\left(\frac{0,0007}{d}+0.00078 \frac{d}{Q_{0}}\right)^{0.25} \cdot \frac{Q_{0}^{2}}{d^{5}} \cdot l
\end{gathered}
$$

\section{Conclusion}

Based on the peculiarities of the system of gas distribution of populated areas, it is advisable to apply such a technical solution, in which gas supply to household consumers, boiler houses, municipal and agricultural enterprises is carried out from the distribution network of high and medium pressure through autonomous combined pressure regulators installed near high-rise buildings.

The gas supply scheme in these cases can be of two variants [15]:

- gas control point with gas measurement, where the pressure is reduced from 1.2 $\mathrm{MPa}$ to 0.3 or $0.6 \mathrm{MPa}$, a single-stage distribution network of medium or high pressure, combined pressure regulators with output low or medium pressure;

- $\quad$ single-stage distribution network with gas supply of $1.2 \mathrm{MPa}$, combined pressure regulators with medium output pressure and combined with them low pressure combined pressure regulators. This scheme makes the gas distribution system very flexible, independent of the connection of any object is achieved. It is possible to connect an additional load. At the same time, metal consumption in distribution networks decreases, due to the reduction in the diameter of the distribution gas pipeline.

The main basic element of the gas distribution scheme is a combined gas pressure regulator that performs the functions of a gas regulator: reducing the gas pressure to a predetermined level, automatically maintaining the gas pressure at a predetermined level regardless of changes in inlet pressure and gas flow, automatic gas shut-off in emergency rises or gas pressure drops after in excess of the set points.

\section{References}

1. M.B. Ravich. Fuel and its efficiency. (Moscow, 1991).

2. K.J. Bell, M.A. Ghaly. Partial Condensers, AICh E Symp. Ser., 69, 72 - 79 (1972).

3. S. Kalchevsky Renewable energy sources, waste energy in industry. Sofia. Avangard Prima (2012).

4. Russian Set of rules 42.101.2003 General provisions for the design and construction of gas distribution systems of metal and polyethylene pipes.

5. Decree of the Government of the Russian Federation of May 14 2013. No. 410.

6. V.A. Zhila, Ye.B. Solovyeva, and M.D. Gulyukin. Nauchnoe obozrenie. 22, 27-32 (2016).

7. Russian State Standard SR 12-529-03 
8. V.A. Zhila, Ye.B. Solovyeva and Yu.G. Markevich. Development of a methodology for determining the optimum reliability indicators for elements of gas supply systems (Moscow, 2016).

9. Ye.B. Solovyeva, N.A. Kharlamova. Nauchnoe obozrenie, 5, 94-98 (2013).

10. Yu.A. Tabunshchikov, V.I. Prokhorov, O.N. Bryukhanov, V.A. Zhila, and A.K. Klochko. Vestnik MSBI, 4, 73-77 (2012).

11. G.M. Kostrova. Requirements for industrial safety at gas distribution and gas consumption facilities. (Moscow, 2016).

12. S.A. Gerelov. Complex system of construction of gas distribution pipelines from polymer materials. (Moscow, 2002)

13. On approval of technical regulations on the safety of gas distribution and gas consumption networks. Decree of the Government of the Russian Federation of October 29 2010. No. 870.

14. GOST 54961-2012.

15. V.A. Zhila. Gas Supply. (Moscow, 2014). 